\title{
Rhadinorhynchus playioscionis n. sp. (Acanthocephala: Rhadinorhynchidae) da pescada (Plagioscion squamosissimus) da Amazônia Brasileira
}

\author{
Vernon E. Thatcher (")
}

\begin{abstract}
Resumo
Rhadinorhynchus plagioscionis $\mathrm{n}$. sp. (Acanthocephala: Rhadinorhynchidae) é descrita do intestino da pescada (Plagioscion squamosissimus (Heckel), obtida perto de Manaus, Amazonas, Brasil. A nova espécie distingue-se das demais espécies do gênero por ter espinhos no tronco com raizes bifurcadas e pontas livres. A distribuição destes espinhos é também diferente, sendo contínua desde o pescoço até a parte posterior aos testículos no macho e bem posterior à campainha uterina na fêmea. A nova espécie tem 12 fileiras de ganchos na probóscide, no sentido longitudinal, com 23 e 24 ganchos por fileira, alternando. No número de ganchos na probóscide. a nova espécie aproxima-se mais da R. trachuri Harada, 1935, de um peixe marinho japonês. R. plagioscionis distingue-se dessa espécie por ter glândulas de cimento que são longas e tubulares (não curtas e em forma de garrafa); lemniscos mals curtos (não mais compridos) que a bainha da probóscide; ovos maiores e um hospedeiro de água doce. A nova espécie é a terceira a ser encontrada em hospedeiro de água doce no mundo.
\end{abstract}

\section{INTRODUÇÃo}

Até o momento, os únicos trabalhos originais sobre os acantocéfalos amazônicos são de Machado Filho (1947), Golvan (1956), Schmidt \& Hugghins (1973) e Thatcher (1979). 0 presente trabalho é o segundo de uma série de estudos a serem feitos dos acantocéfalos da região. Um representante do gênero Rhadinorhynchus foi citado anteriormente de águas marinhas brasileiras, segundo Cable \& Linderoth (1963).

\section{MÉtodos e materiais}

Os hospedeiros foram comprados de um peixeiro em Manaus, Amazonas, Brasil. Os vermes foram retirados do trato digestivo, colocados em água, para provocar a extensão da probóscide, e logo fixados em álcool-formolácido acético (AFA). Depois de serem lavados em água para remover o fixador, a cutícula foi furada para permitir a desidratação completa, e facilitar a coloração. Foram corados pelo Carmalum de Mayer, desidratados numa série de álcool, clarificados em salicilato de metila e montados no bálsamo do Canadá. Os desenhos foram feitos com a ajuda de uma câmara clara e as medições com uma ocular micrométrica. Entre as medições, os limites superiores referem-se ao holótipo e alótipo, respectivamente, e os inferiores foram tomadas do menor casal entre os parátipos. Todas as medidas são dadas em milímetros, exceło as dos ovos e ganchos que são em micra.

\section{SEÇÃO SISTEMÁTICA}

RHADINORHYNCHIDAE Travassos, 1923

RHADINORHYNCHINAE Lühe, 1911

Rhadinorhynchus Lühe, 1911 (emend. Cable e Linderoth, 1963)

Rhadinorhynchus plagioscionis $\mathrm{n}$. sp. (Fig. 1 - 12)

Hospedeiro: Plagioscion squamosissimus (Heckel) "pescada".

Habitat: Intestino.

Procedência: Manaus, Amazonas, Brasil.

Holótipo e Alótipo: Instituto Nacional de Pesquisas da Amazônia (INPA).

Parátipos: INPA e Museu de Zoologia da Universidade de São Paulo.

Diagnose específica (baseada em 8 exemplares e medições de 4): Tronco cilíndrico; diâmetro máximo pré-equatorial; diâmetro diminui ligeiramente para as extremidades; curvado dorsalmente. Probóscide alongada, curvada ventralmente; com ganchos maiores $\mathrm{e}$ mais largos no lado ventral. Ganchos na probóscide arranjados quincuncialmente; com 12 fileiras longitudinais; cada fileira com 23 ou 24 ganchos, números alternados. Os ganchos mais compridos se encontram no fim do terço

(•) - Instituto Nacional de Pesquisas da Amazónia, Manaus. 


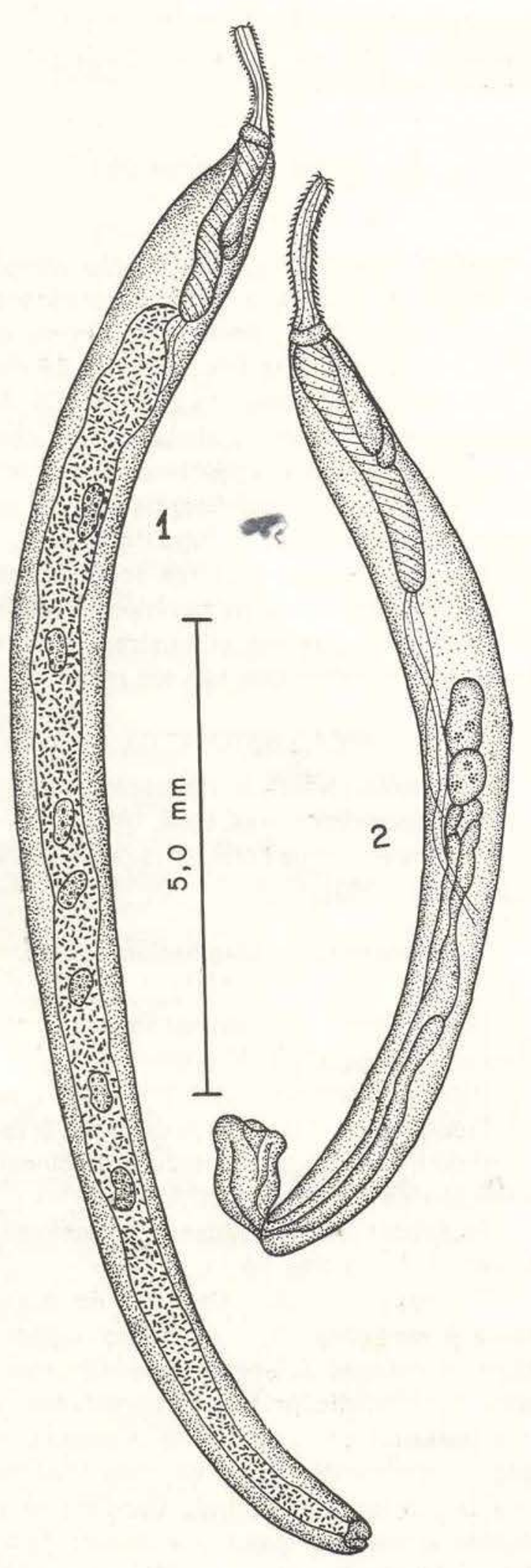

Fig. 1-2-Rhadinorhynchus plagioscionis $\mathrm{n}$. $\mathrm{sp}$. : 1) fêmea adulta, vista lateral; 2) - macho adulto, vista lateral.

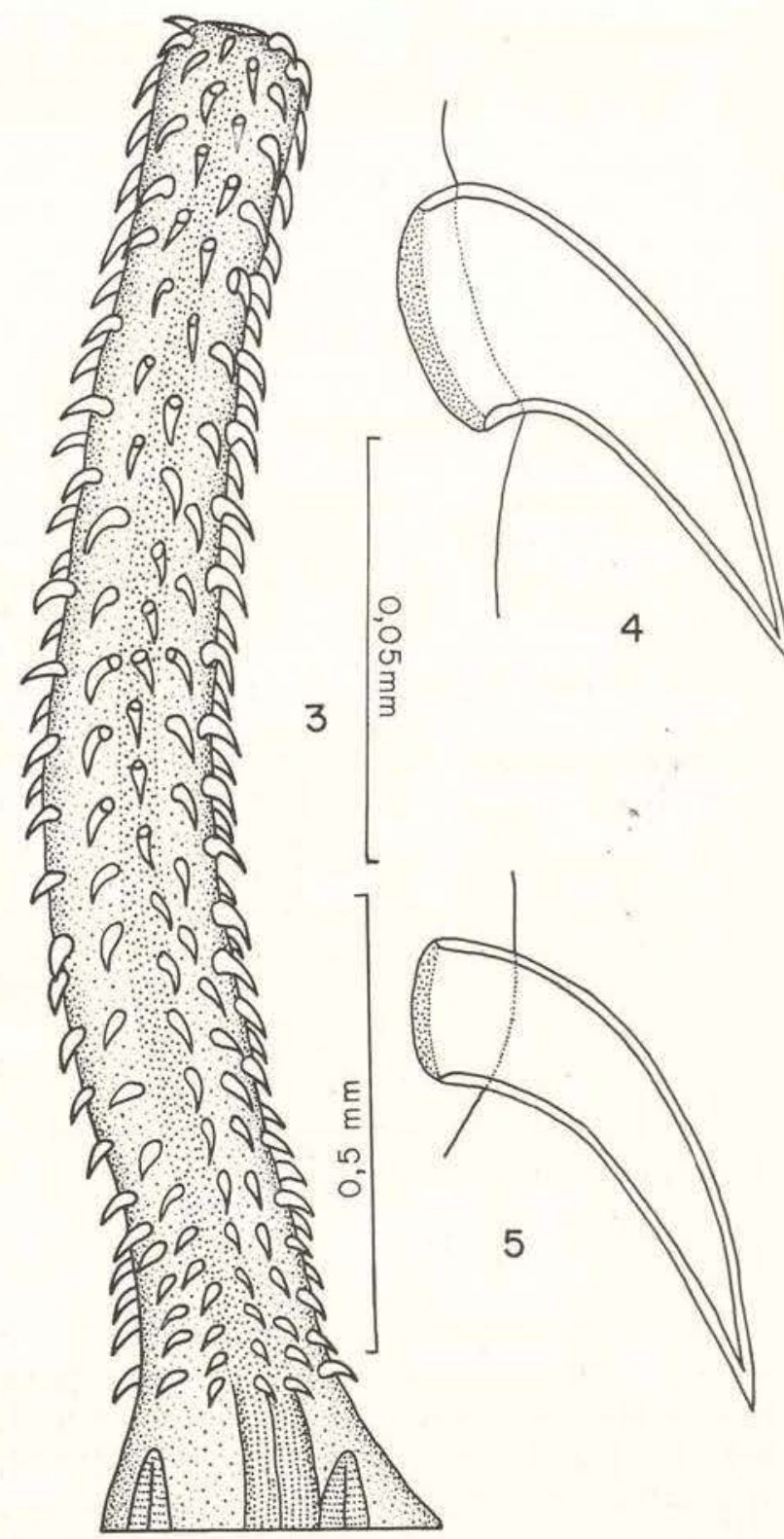

Fig. 3-5 - Rhadinorhynchus plagioscionis n. sp.: 3) probóscide, vista lateral; 4) - gancho ventral da probóscide; 5) - Gancho dorsal da probóscide.

anterior da probóscide; reduzem-se ligeiramente na ponta e gradualmente para a base. Os ganchos menores encontram-se no último círculo da base. Bainha da probóscide com parede dupla. Fêmea maior que o macho. Gânglio central no meio da probóscide. Lemniscos ligeiramente desiguais.

Macho: Tronco medindo $8,8-11,0$ de comprimento e 0,73-1,05 de diâmetro máximo ao nível da porção posterior da bainha, Espinhos no tronco distribuídas quincuncialmente des- 


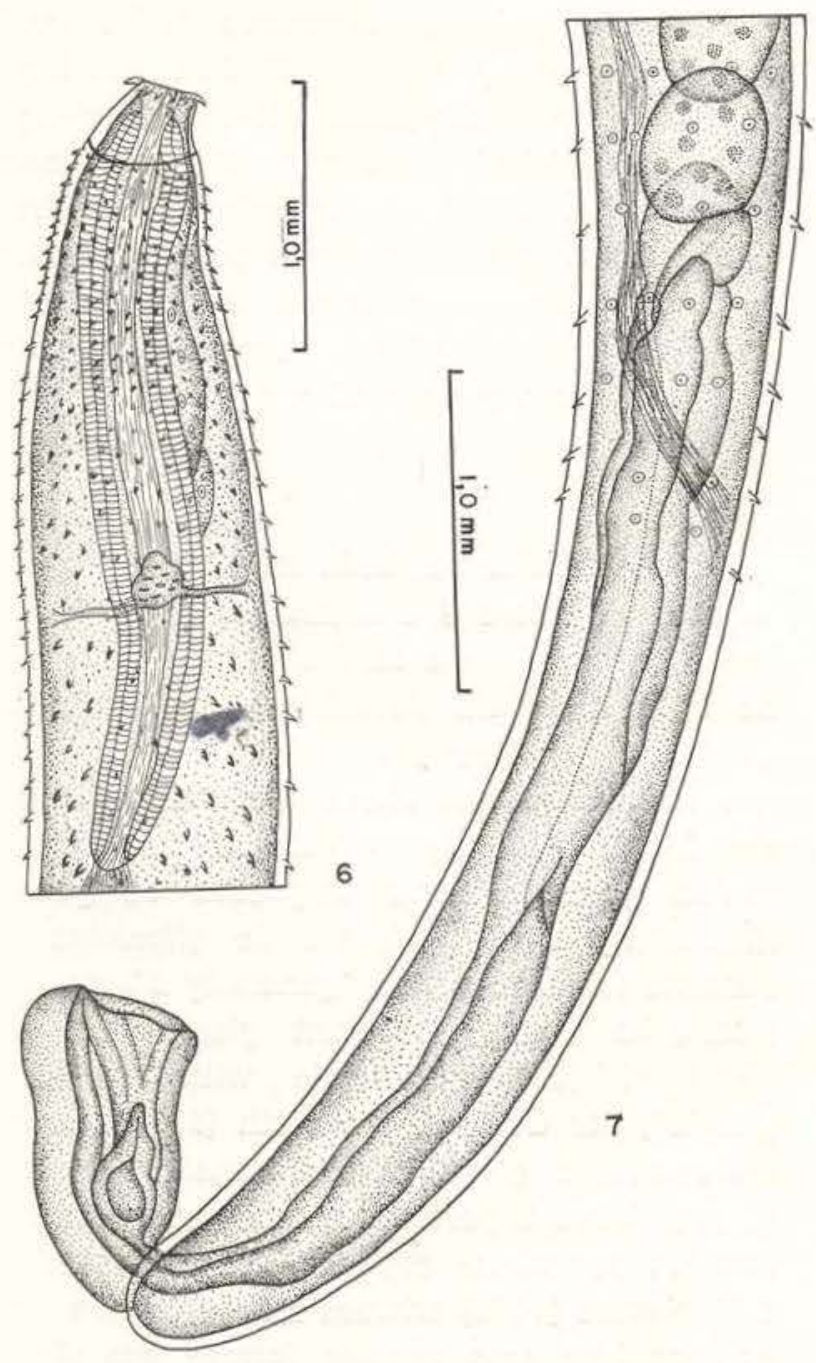

Fig. $6 \cdot 7-$ Rhadinorhynchus plagioscionis $\mathrm{n} . \mathrm{sp} .: 6$ ) Regiăo anterior do tronco, mostrando pescoço, bainha da probóscide, lemniscos, gânglio central e espınhos; 7) - Região posterior do macho, mostrando testículos, glândulas de cimento e bolsa copuladora.

de o pescoço até posterior aos testículos; mais numerosos ventral e anteriormente; com raízes bifurcadas e pontas projetando; medem $12-14 \times 17-24 \mu \mathrm{m}$. Probóscide mede 1,52-1,58 de comprimento e 0,17 de diâmetro. Ganchos da probóscide de três formas, sendo os ventrais mais compridos e mais largos; ganchos ventrais com $23 \times 69 \mu \mathrm{m}$, tamanho máximo; ganchos dorsais com 12-20 $\times 57 \mu \mathrm{m}$, tamanho máximo; ganchos basais com $12-17 \times 24-46 \mu \mathrm{m}$. Pescoço mede $0,15-0,20$ de comprimento e $0,32-0,38$ de largura. Bainha da probóscide mede 2,75-3,15 de comprimento e 0,38-0,42 de diâmetro máximo. Lemnisco maior mede 1,30-1,65 de comprimento e $0,13-0,20$ de largura. Lemnisco menor mede $1,20-1,60$ de comprimento e $0,13-0,20$ de largura. Sistema reprodutor ocupa $63-68 \%$ do comprimento do tronco. Testículo anterior o maior; mede $0,42-0,90$ de comprimento e 0,28-0,47 de diâmetro. Testículo posteriar mede 0,36-0,66 de comprimento e 0,28-0,45 de diâmetro. Glândulas de cimento 4; longos, tubulares; 2 atingem o testículo posterior e os outros 2 são ligeiramente mais curtos. Bolsa copuladora mede $0.75 \times 1,42$.

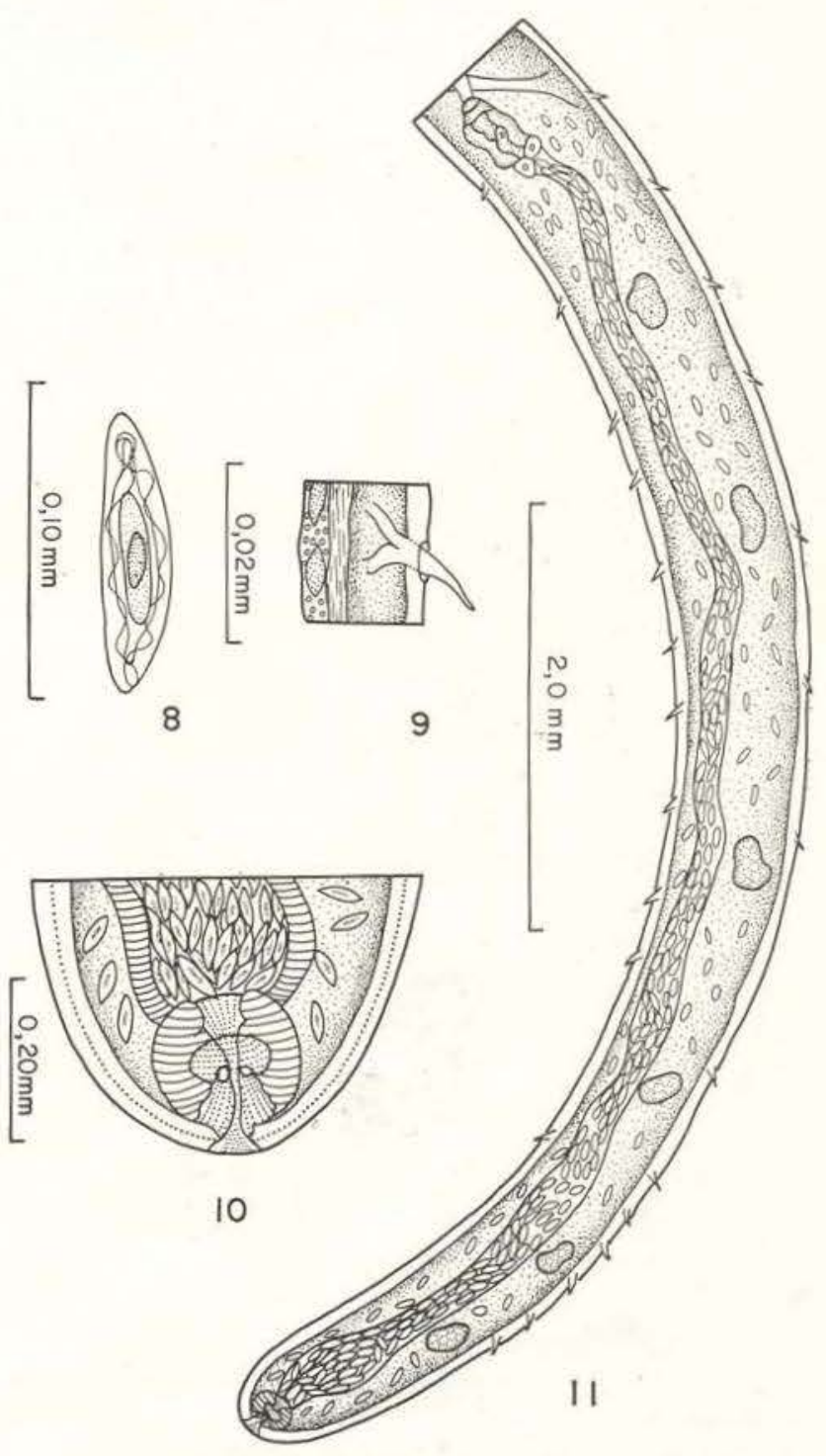

Fig. 8-11-Rhadinorhynchus plagioscionis n. sp.: 8) Ovo, mostrando as três cascas e os filamentos; 9) Espinho cuticular com raíz bifurcada; 10) - Extremidade posterior da fêmea, mostrando útero, vagina e esfinter vaginal; 11) - Região posterior da fêmea, mostrando o comprimento do útero, os ovários e a campainha uterina. 
Fêmea: Tronco mede 14,5-16,4 de comprimento e $0,85-1,0$ de diâmetro. Espinhos no tronco estendem-se mais posteriormente na fêmea que no macho; medem $12 \times 19-24 \mu \mathrm{m}$. Probóscide parecida com a do macho; mede 1,52-1,60 de comprimento e 0,15-0,17 de diâmetro. Ganchos ventrais com $23 \times 69 \mu \mathrm{m}$, tamanho máximo; dorsais com 12-14 x 65-69 $\mu \mathrm{m}$, tamanho máximo; ganchos basais com 10-14 $x$ 44-46 $\mu \mathrm{m}$. Pescoço mede 0,22 de comprimento e 0,32 de diâmetro máximo. Bainha da probóscide mede 2,7-2,8 de comprimento e 0,32

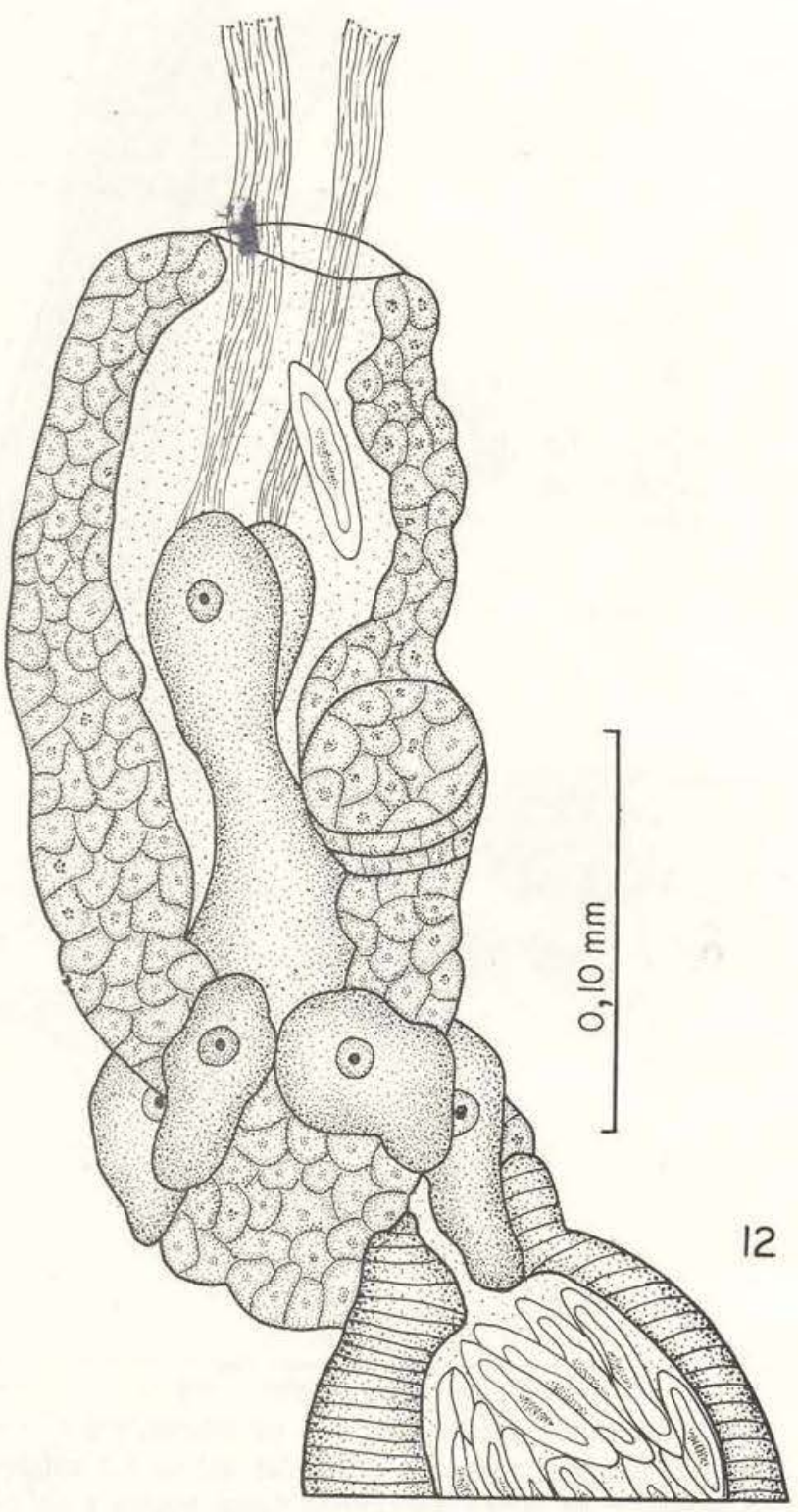

Fig. 12 - Rhadinorhynchus plagioscionis n. s.: Campainha uterina. de diâmetro máximo. Lemnisco maior mede $1,50-1,60$ de comprimento e $0,16-0,18$ de largura. Lemnisco menor mede $1,47-1,55$ de comprimento e $0,16-0,18$ de largura. Sistema reprodutor ocupa $42-53 \%$ do comprimento do tronco. Campainha uterina mede aproximadamente 0,25 de comprimento e 0,10 de diâmetro. Ovos com prolongações e filamentos na casca mediana. Ovos medem $23 \times 110 \mu \mathrm{m}$.

\section{Discussão}

Existe muita confusão na literatura quanto a este gênero e à família Rhadinorhynchidae. Em parte, esta confusão foi o resultado da dificuldade em determinar o número de glândulas de cimento do macho e do fato de que várias espécies foram descritas baseadas em fêmeas, impossibilitando a contagem daquelas glândulas. Nas diagnoses do gênero Rhadinorhynchus, o número de glândulas de cimento foi dado como, "generally 8 ", por Petrochenko (1956); "cement glands 2", por Yamaguti (1963); e "male with 4 cement glands", por Cable \& Linderoth (1963). O número destas glândulas foi considerado uma característica importante para a separação das famílias por várias especialistas. Van Cleave \& Lincicome (1940) criaram a nova família Gorgorhynchidae para aquelas formas que têm 4 glândulas e deixaram as formas com 8 dentro da Rhadinorhynchidae. Cable \& Linderoth (1963) rejeitaram este conceito porque, segundo eles, o número das glândulas não tem tanta importância e a espécie tipo de Rhadinorhynchidae só tem 4 glândulas. Apesar disso, Golvan \& Houin (1964) publicaram uma revisão da família Gorgorhynchidae Van Cleave \& Lincicome, 1940, incluindo nela várias espécies anteriormente consideradas como representantes de Rhadinorhynchus. Concordo com a interpretação de Cable \& Linderoth (1963) e como o presente material tem 4 glândulas de cimento, e com parte a maioria das características com o gênero por eles redefinido, é apropriado colocar esta nova espécie no Rhadinorhynchus, família Rhadinorhynchidae.

Rhadinorhynchus plagioscionis $\mathrm{n}$. $\mathrm{sp}$. distingue-se das demais espécies do gênero pela forma e distribuição dos espinhos no tron- 
co. As outras espécies do gênero têm os espinhos só na porção anterior do tronco e são freqüentemente divididas em dois grupos. Ao contrário, a nova espécie tem espinhos cobrindo uma área contínua que se estende posteriormente. R. plagioscionis tem espinhos com raízes bifurcadas e pontas livres, mas as outras espécies têm espinhos com raízes simples e pontas cobertas de cutícula. Quanto ao número de ganchos na probóscide, a $R$. plagioscionis $\mathrm{n}$. sp. aproxima-se mais da espécie $R$. trachuri Harada, 1935, a qual foi citada dum peixe marinho no Japão. $R$. plagioscionis distingue-se desta por ter: os ganchos mais posteriores da probóscide menores (não maiores) aos outros ganchos; lemniscos menos compridos que a bainha da probóscide (não mais compridos); glândulas de cimento compridas e tubulares (não curtos e em forma de garrafa); Ovos maiores e um hospedeiro de água doce. Mais duas espécies de Rhadinorhynchus foram citadas de peixes de água doce. Uma delas encontra-se infectando as carpas da China e a outra procede do rio Nilo. mas a nova espécie, aqui descrita, não tem características em comum com aquelas espécies.

\section{AgRAdeCIMENTOS}

Agradeço à desenhista, Srta. Maria de Fátima Vieira, pela ajuda prestada na preparação dos desenhos.

\section{SUMMARY}

Rhadinorhynchus plagioscionis $\mathrm{n}$. $\mathrm{sp}$. (Acanthocephala: Rhadinorhynchidae) is described from the intestine of a freshwater fish called the "pescada" (Plagioscion squamosissimus (Heckel) from Manaus, Amazonas, Brazil. The new species differs from others in the genus in that the trunk spines have bifurcated roots and free points. The distribution of these spines is also different, being from the neck region posteriorly to beyond the testes in the male, and well-posterior to the uterine bell in the female. The new species has 12 longitudinal rows of hooks on the proboscis, quincuncially arranged, with 23 and 24 hooks per row alternating. In hook number, the new species most resembles R. trachuri Harada, 1935, described from a
Japonese marine fish. R. plagioscionis differs from that species, however, in having cement glands that are long and tubular (not short and flask-shaped); lemnisci that are shorter (not longer) than the proboscis sac; larger eggs and a freshwater host. The new species is the thir in the genus to be reported from a freshwater host fish in the world.

\section{BIBLIOGRAFIA}

CABLE, R.M. \& LINDEROTH, J.

1963 - Taxonomy of some Acanthocephala from marine fishes with reference to species from Curaçao, N.A., and Jamaica W.I. J. Parasit., $49(5): 706-716$.

Golvan, Y.J.

1956 - Acanthocéphales d'Amazonie. Redescription d'Oligacanthorhynchus iheringi Travassos, 1916 et description de Neoechinorhynchus buttnerae $n$. sp. (Neoacanthocephala-Neoechinorhynchidae). Ann. Parasit. Hum. Comp., 31(5) : 500-524.

Golvan, Y.J. \& Hounn, R.

1964 - Revision des Palaeacanthocephala (Deixième Note), La Famille des Gorgorhynchidae Van Cleave et Lincicome 1940. Ann. Parasit. Hum. Comp., 39(5) : 535-605.

Machado Filho, D.A.

1947 - Revisão do gênero "Polyacanthorhynchus" Travassos, 1920 (Acanthocephala, Rhadinorhynchidae). Rev. Brasil. Biol., $7(2)$ : $7(2): 195-201$.

Petrochenko, V.I.

1956 - Acanthocephala of Domestic and Wild Animals (tradução do ruso, 1971). Keter Press. Jerusalem, Israel. p. $1-465$ (Vol. I.).

Schmid, G.D. \& Hugghins, E.J.

1973 - Acanthocephala of South American fishes. Part I, Eoacanthocephala. J. Parasit., 59(5) : 829-835.

Thatcher, V.E.

1979 - Uma nova espécie de Gorytocephalus Nickol e Thatcher, 1971 (Acanthocephala: Neoechinorhynchidae) do acari bodó (Pices: Loricariidae) da Amazônia, Brasil. Acta Amazonica, 9(1) : 199-202.

Van Cleave, H.J. \& Lincicome, D.R.

1940 - A reconsideration of the acanthocephalan family Rhadinorhynchidae. J. Parasit., 26(1): 75-81.

YAMAGUTI, $\mathrm{S}$.

1963 - Sistema Helminthum, Vol. V., Acanthocephala. Intersci, Pub. New York. 423 p.

(Aceito para publicação em $9 / 6 / 80$ ) 